\title{
Gene and cell therapy in South Africa: Current status and future prospects
}

\author{
A Ely, ${ }^{1}$ BSc, BSc(Hons), MSc, PhD; K Bloom, ${ }^{1}$ BSc, BSc(Hons), MSc, PhD; M B Maepa, ${ }^{1}$ BSc, BSc(Hons), MSc, PhD; \\ J N Mahlangu, ${ }^{2}$ BSc, MB BCh, FCPath (Haem), MMed; M S Pepper, ${ }^{3}$ MB ChB, PhD, MD, PD; P Arbuthnot, ${ }^{1}$ MB BCh, PhD \\ ${ }^{1}$ Wits/SAMRC Antiviral Gene Therapy Research Unit, Faculty of Health Sciences, University of the Witwatersrand, Johannesburg, South Africa \\ ${ }^{2}$ Haemophilia Comprehensive Care Centre, Faculty of Health Sciences, University of the Witwatersrand and National Health Laboratory Service, \\ Johannesburg, South Africa \\ ${ }^{3}$ SAMRC Extramural Unit for Stem Cell Research and Therapy and Institute for Cellular and Molecular Medicine, Department of Immunology, \\ University of Pretoria, South Africa
}

Corresponding author: A Ely(abdullah.ely@wits.ac.za)

\begin{abstract}
South Africa has a high disease burden resulting from communicable and non-communicable diseases. Current therapeutic interventions rarely result in a cure and the associated lifelong treatment places a considerable strain on an overburdened health sector. Gene and cell therapies present novel alternatives to disease management, offering the promise of a single treatment and a lifelong cure. Although challenges remain, investment in the field has started to bear fruit, with a number of gene and cell therapeutics reaching the market in the past decade. To take full advantage of these developments, it is important that a proactive approach to nurturing appropriate human and material resources is adopted in the country.
\end{abstract}

S Afr Med J 2019;109(8 Suppl 1):S13-S17. https://doi.org/10.7196/SAMJ.2019.v109i8b.13819

The use of DNA as a drug was first proposed with the advent of molecular biology. ${ }^{[1,2]}$ Advances in our understanding of the genetic basis of disease, coupled with progress in manipulating DNA, gave rise to the concept of genetic modification of an organism to correct a diseased state. Monogenic disorders offered convenient model systems to test this concept, as pathologies can be ascribed to abnormalities of single genes and replacement of aberrant genes with functional ones would, theoretically, reverse the pathology. As early as the 1970s, researchers explored ways of introducing DNA into cells to produce a therapeutic effect. ${ }^{[1,3]}$ The first trial to evaluate genetic modification in humans employed the Shope papillomavirus (SPV) to correct hyperargininaemia, an inherited disorder that results from arginase deficiencies. SPV was thought to carry a copy of the arginase gene and it was proposed that infection with this virus would restore activity of the enzyme and reduce plasma arginine levels. ${ }^{\left[{ }^{[3]}\right.}$ The virus was produced in large quantities, purified and administered to three siblings with hyperargininaemia. The trial proved unsuccessful, which the authors attributed to degeneration of the virus. However, subsequent research showed that SPV does not carry an arginase gene. ${ }^{[4]}$ Although the trial yielded less-than-desirable results, the potential of using a virus as a vector for the delivery of therapeutic genes to cells was a revolutionary concept.

Emergence of recombinant DNA technology marked a turning point in the field of gene therapy. The ability to isolate and copy genes presented the way forward to produce functional copies of genes that are required for replacement therapy. This was the approach of the first approved human trial that evaluated delivery of a therapeutic gene. ${ }^{[5]}$ The study aimed to correct severe combined immunodeficiency (SCID) resulting from a mutation of the gene encoding the adenosine deaminase (ADA) enzyme. Blaese et al. ${ }^{[5]}$ cloned a functional copy of the ADA gene and inserted it into a replication-defective retrovirus using recombinant DNA technology. T cells were harvested from two patients with ADA-SCID, transduced with the recombinant virus and reinfused into the patients. The trial was carried out in 1990 and was hailed a success owing to the positive response seen in one of the two recipients. However, the field suffered a considerable setback in 1999, when a patient, Jesse Gelsinger, died after receiving gene replacement therapy in an efficacy trial aimed at restoring ornithine transcarbamylase deficiency. ${ }^{[6]}$

Since these initial exploratory studies, the field has grown to encompass a variety of therapeutic modalities that extend beyond gene replacement therapy for monogenic disorders. An advantage of gene therapy drugs is that their design is centred on rational principles. The genomics era has granted researchers unprecedented insight into the genetics underlying disease, and with the use of easy-to-understand principles it is possible to rationally design gene therapies to correct disease at a molecular level. In the modern era, gene therapy can be defined as the use of any nucleic acid sequence either DNA or RNA - to produce a therapeutic effect, and therefore takes many forms. It may entail the use of a gene that undergoes transcription and translation to produce a therapeutic RNA or protein. Alternatively, the nucleic acid sequence itself may carry out the therapeutic function by binding (e.g. aptamers) or modifying (e.g. DNAzymes or ribozymes) disease targets.

Gene therapy drugs typically comprise the therapeutic nucleic acid sequence contained within a delivery vector (Fig. 1). However, gene therapy has also given rise to cell therapy, which deals with the transfer of intact therapeutic cells to patients. This typically entails using technologies of gene therapy to engineer cells that confer some therapeutic characteristic. Advances in the fields of molecular biology, genetics and microbiology, among others, have driven progress in gene and cell therapy, and have facilitated the development of safer and increasingly efficacious therapeutics. The rapid development in the field is highlighted by the large number of clinical trials underway worldwide $^{[7]}$ and the recent approval of four gene-based therapeutics by the US Food and Drug Administration (FDA). ${ }^{[8]}$ 
A

\section{dovarer}

Therapeutic gene (in viral genome)

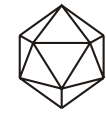

Recombinant adeno-associated virus
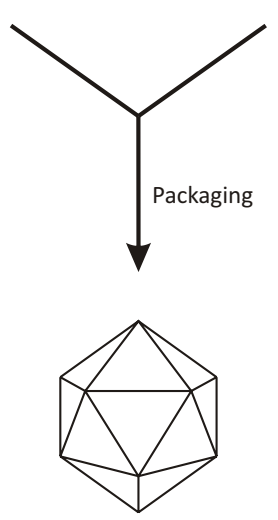

Recombinant adenovirus

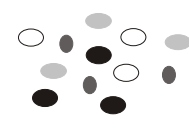

Virus assembly components

B

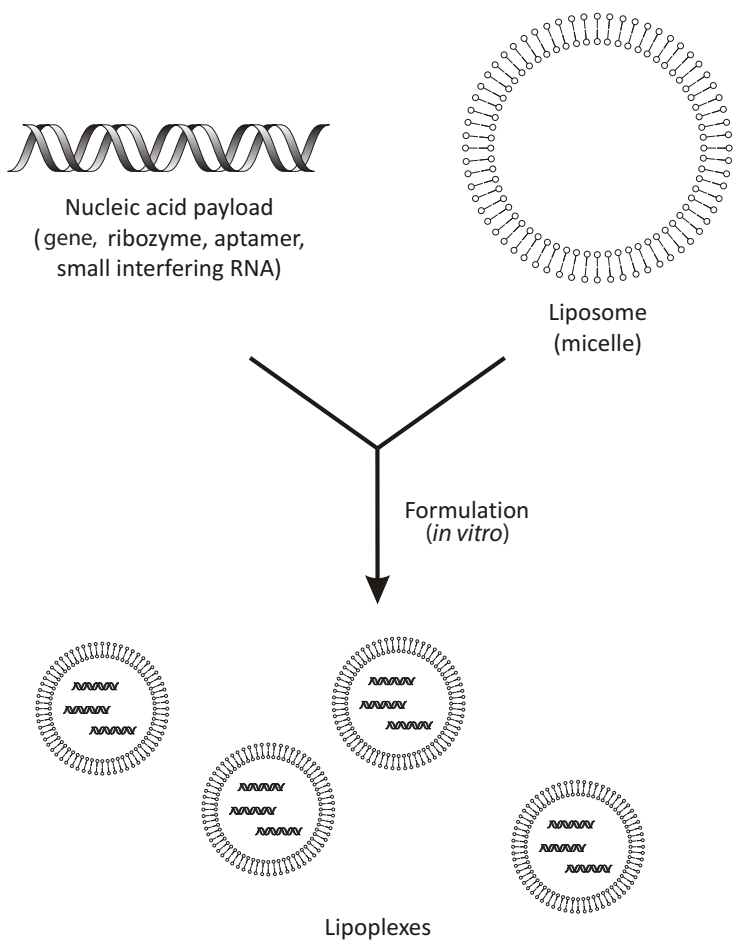

Recombinan retrovirus

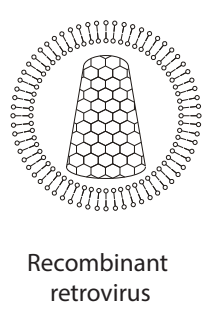

Lipoplexes

Fig. 1. Gene therapy technologies in the modern era. Gene therapies typically comprise the therapeutic sequence complexed with or incorporated into a vector for delivery. Delivery vectors are broadly divided into viral (A) and non-viral (B) vectors. Viruses are efficient at delivering their nucleic acid payload and are popular vectors for delivery of gene therapy. Recombinant viral vectors are produced by exploiting the virus' natural assembly process. Typically, a recombinant viral genome that contains the therapeutic gene is expressed with viral structural and assembly components in a packaging cell line. The recombinant viral genome is subsequently assembled into the recombinant vector and can then be harvested and purified. As these vectors exploit the process of viral replication, the incorporated therapeutic sequences have to be a gene. In contrast, non-viral vectors can accommodate any nucleic acid sequence. Liposomes are the most common form of non-viral vector used in gene therapy applications and exploit the ability of lipids to form micelles in an aqueous environment. As these micelles share the same chemistry as the lipid bilayer of cells, they are able to fuse with it and so deliver their cargo into the cytoplasm. The use of cationic lipids in liposome formulation has proven to be ideal as it allows interaction not only with the negatively charged cell membrane but also with its negatively charged nucleic acid payload.

To date, gene and cell therapy research in South Africa (SA) has been limited to laboratory-based experimental work and preclinical studies in mice. While there has not been a clinical trial initiated in the country, several haemophilia gene therapy programmes have been established in SA as an extension of existing international trials. On the continent as a whole, six gene therapy trials are currently underway or have received approval, but account for only $0.2 \%$ of the total number of trials worldwide. ${ }^{[7}$

The toolbox of strategies available to gene and cell therapy is continually expanding and currently includes gene replacement therapy, gene silencing and gene editing (Table 1). Discoveries in the biological sciences that have possible therapeutic application are rapidly being applied in the field of gene therapy. For example, RNA interference (RNAi) was being explored as a gene therapy approach soon after its discovery. The pathway uses RNA sequences called microRNAs to regulate gene expression. Artificial microRNAs can be designed to exploit this pathway and suppress any gene of interest and have been used extensively to silence pathology-causing genes or genes required for viral replication. Similarly, research in bacterial systems has revolutionised gene therapy and ushered in the era of genome editing. Transcription activator-like effector nucleases (TALENs) and the clustered regularly interspaced short palindromic repeats (CRISPR) and the CRISPR-associated (Cas) protein system are actively being assessed for their potential to disable viruses, correct genetic mutations or engineer cell therapies.

SA and the rest of the continent suffer from a considerable disease burden, and while gene and cell therapy approaches may not be a panacea, they do offer novel avenues to be explored for treating intractable diseases such as HIV/AIDS.

\section{Gene and cell therapy in South Africa Concerning delivery}

An important consideration for the use of gene therapy is the vector employed to deliver the therapeutic sequence to the intended site of action. Unlike small-molecule drugs, the large size and negative charge of nucleic acids make these molecules inherently refractory to crossing the negatively charged cell membrane. Viruses are naturally effective at delivering their genomes to host cells and are an obvious choice for delivery of therapeutic genes to tissues in vivo. To limit adverse effects related to the use of these vectors, they have been re-engineered by removing genes required for viral replication. Viral vectors are therefore replication defective and, while retaining their ability to infect cells and deliver their nucleic acid payload, are incapable of spreading. Recombinant viruses remain the vectors of 


\begin{tabular}{|c|c|c|}
\hline Disease type & Gene therapy & Cell therapy \\
\hline $\begin{array}{l}\text { Monogenic disorders (e.g. } \\
\text { haemophilia A and B) }\end{array}$ & $\begin{array}{l}\text { Gene replacement therapy } \\
\text { - Delivers a functional copy of the mutant gene to the target } \\
\text { organ } \\
\text { - Licensed gene replacement therapies: Glybera, Strimvelis } \\
\text { Gene repair } \\
\text { - Repairs mutant gene in situ using gene-editing technologies } \\
\text { (zinc finger nucleases, TALENs, CRISPR/Cas) }\end{array}$ & Not applicable \\
\hline Cancer & $\begin{array}{l}\text { Suicide gene therapy } \\
\text { - Delivers suicide gene to the tumour } \\
\text { - Expression of suicide gene produces a toxic compound that } \\
\text { kills the tumour } \\
\text { - Example: Imlygic } \\
\text { Anti-oncogene therapy } \\
\text { - Oncogene expression inhibited with therapeutic DNA or RNA } \\
\text { sequences } \\
\text { - Example: RNA interference-mediated silencing } \\
\text { Tumour suppressor gene therapy } \\
\text { - Delivers tumour suppressor gene }(p 53) \text { to tumour } \\
\text { - Example: Gendicine }\end{array}$ & $\begin{array}{l}\text { Chimeric antigen receptor } \mathrm{T} \text { cells } \\
\text { - } \mathrm{T} \text { cells are modified to express a } \mathrm{T} \text { cell } \\
\text { receptor (TCR) targeted to specific } \\
\text { tumour antigens } \\
\text { - Viral and non-viral vectors used to deliver } \\
\text { TCR gene to } \mathrm{T} \text { cells ex vivo } \\
\text { - Modified } \mathrm{T} \text { cells transplanted into the } \\
\text { patient } \\
\text { - Licensed therapies: Kymriah, Yescarta }\end{array}$ \\
\hline Infectious diseases & $\begin{array}{l}\text { Gene silencing } \\
\text { - Viral genes silenced with therapeutic DNA or RNA } \\
\text { - Host genes required for viral replication silenced } \\
\text { - Example: Exploiting the RNA interference pathway to silence } \\
\text { genes } \\
\text { Genome editing }\end{array}$ & $\begin{array}{l}\text { Ex vivo therapy } \\
\text { - Patient cells depleted of host-dependency } \\
\text { factor ex vivo } \\
\text { - Resistant cell reinfused into the patient } \\
\text { - Potential of this strategy highlighted by } \\
\text { the Berlin patient, Timothy Brown (HIV) }\end{array}$ \\
\hline
\end{tabular}

TALENs = transcription activator-like effector nucleases; CRISPR/Cas = clustered regularly interspaced short palindromic repeats/CRISPR-associated protein system .

choice for gene and cell therapy, accounting for $70 \%$ of all clinical trials. $^{[7]}$

Retroviral vectors were the first to be used and excellent successes were achieved in correcting SCID. However, concerns regarding the oncogenic potential of these viruses have led to a decline in their use. With lower oncogenic potential, lentiviruses are considered safer than other retroviruses and are still commonly used for gene therapy approaches. To date, adenoviruses have proven to be the most popular viral vector as they can accommodate large genes, are efficient delivery agents and achieve high levels of transgene expression. However, potent immune stimulation by adenoviruses is a major limitation of their use, as illustrated by the death of Jesse Gelsinger, ${ }^{[6]}$ which has been attributed to a severe immune reaction to the recombinant adenovirus he received. In more recent years adeno-associated viruses (AAVs) have gained in popularity. ${ }^{[9,10]}$ Although the AAV genome is relatively small, which limits the size of the therapeutic genes that these vectors can accommodate, AAVs are widely considered to be non-pathogenic and can infect a wide range of cell types at high efficiencies. More importantly, AAVs have been shown to express therapeutic sequences over the long term, a key consideration when developing gene therapy for chronic conditions.

The development of non-viral vectors (particularly cationic liposomes ${ }^{[11]}$ ) has offered an alternative to viral vectors for delivering therapeutic sequences. Non-viral vectors offer a number of advantages over viral vectors, which include: $(i)$ an improved safety profile, as they are non-immunogenic; (ii) facile and inexpensive up-scale of manufacture compared with viral vectors, and (iii) the ability to accommodate any type of nucleic sequence and not only genes. However, the use of non-viral vectors involves unique challenges, which include optimising serum stability in vivo, targeted delivery to sites of disease and endosomal escape upon cell entry. The Non-Viral Gene Delivery Laboratory at the University of KwaZulu-Natal, SA, focuses on the development of cationic lipids for the formulation of hepatotropic liposomes. Using fundamental principles of biochemistry, this group has produced promising results with regard to the design of liposomes that exhibit enhanced stability, targeted delivery to liver-derived cells and escape from endolysosomal degradation. ${ }^{[12-14]}$ This group has also evaluated the use of gold nanoparticles as synthetic vectors for the delivery of DNA drugs. The data from studies on cultured cells are promising and the technology offers value for clinical translation. Limitations of delivery vectors remain the most important hurdle to be overcome before widespread use of gene therapy becomes a clinical reality. However, continuous development of both viral and non-viral vectors is advancing the field to the point where clinical translation of gene therapy will be routine.

\section{Gene and cell therapy against HIV infection}

The scourge of the HIV/AIDS pandemic has been exacerbated by none of the available interventions being able to cure the disease. However, gene or cell therapy offers hope in this respect. A distinction should be made between a sterilising cure and a functional cure. A sterilising cure results not only in an undetectable viral load but also in the elimination of the viral reservoir. The well-publicised case of Timothy Brown is the only reported case of a sterile cure. ${ }^{[6]}$ In contrast, a functional cure would result in a reduced or absent viral load, but not in eliminating the viral reservoir. In this case, a reduced requirement for antiretroviral therapy and a less-compromised 
immune system could prove potentially beneficial, as it may result in fewer infections and better immune surveillance (and possibly also fewer cancers). The objective of the South African Medical Research Council (SAMRC) Extramural Unit for Stem Cell Research has been to identify and target host elements in order to render $\mathrm{CD} 4^{+} \mathrm{T}$ cells resistant to HIV ${ }^{[15]}$ This was achieved by targeting expression of C-C chemokine receptor type 5, a co-receptor for viral entry, using an artificial microRNA. ${ }^{[16]}$ This gene-engineering approach was assessed in vivo in human immune system (HIS) mice infected with HIV as a preclinical proof of concept. ${ }^{[17]}$ Work was also directed at optimising the ex vivo expansion of haematopoietic stem cells. ${ }^{[18]} \mathrm{Key}$ findings and expertise gained relate to: (i) efficient delivery of the lentiviral construct into haematopoietic stem and progenitor cells (HSPCs); (ii) demonstration of multilineage haematopoiesis from gene-engineered HSPCs, and (iii) functional cure of HIV when HIS mice were transplanted with gene-engineered HSPCs (preclinical proof of concept). The group is working towards initiating a phase I clinical trial based on the preclinical data.

\section{Gene therapy for viral hepatitis}

Infection with the hepatitis B virus (HBV) remains a serious medical problem, with $\sim 257$ million chronic patients worldwide. ${ }^{[19]}$ Sub-Saharan Africa is a region of high endemicity, with a reported burden of $4.6-8.5 \%$. Chronic infection is associated with cirrhosis and hepatocellular carcinoma, which together were responsible for $\sim 867000$ deaths worldwide in 2016. The limited benefit of available intervention strategies has led the Wits/SAMRC Antiviral Gene Therapy Research Unit (AGTRU) to explore gene therapy against chronic HBV infection. The group has a well-established workflow, from basic development to taking therapeutics to preclinical testing in animal models. Leveraging its expertise in molecular biology, the unit has been able to capitalise on developments in gene therapy to create novel antiviral therapeutics. The AGTRU has published extensively on RNA-based therapeutics, including ribozymes, small interfering RNAs and artificial microRNAs. ${ }^{[20-22]}$ More recently the unit has been exploring the use of TALENs ${ }^{[23]}$ and the CRISPR/Cas system $^{[10]}$ for targeted mutation of HBV DNA, which disables the virus. While this approach does not achieve a sterilising cure, it is able to produce a functional cure for hepatitis $\mathrm{B}$, a previously undreamt of goal in anti-HBV therapeutics. The unit has also explored the use of recombinant adenoviruses, ${ }^{[24]}$ lentiviruses ${ }^{[25]}$ and AAVs, ${ }^{[9,10]}$ given its core competency in viral vectorology. Considerable progress has been made and a number of candidates have been identified for further development. Current research efforts are directed at clinical translation

\section{Haemophilia gene therapy}

Haemophilia $\mathrm{A}$ and $\mathrm{B}$ are $\mathrm{X}$-linked monogenic disorders caused by deficiencies in coagulation factor VIII (FVIII) and IX (FIX), respectively. ${ }^{[26]}$ The epidemiology and natural history of haemophilia are mirrored in all nations and geographic regions of the world, including developing countries, where haemophilia is grossly underdiagnosed and poorly managed. Deficiency of FVIII or FIX presents with spontaneous or trauma-induced bleeding, predominantly into joints, with consequent crippling and debilitating

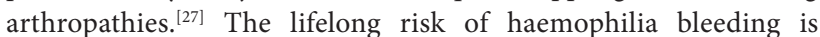
associated with high mortality and significant morbidity. The current standard of care for haemophilia uses plasma-derived or recombinant products that replace the missing factor. There are several challenges associated with replacement therapy, including patients' developing antibodies against the replacement proteins and the increased treatment burden associated with regular intravenous infusion of clotting factors. ${ }^{[28]}$ Inhibitors of replacement therapy are found in up to $30 \%$ of patients and result in poor quality of life, increased mortality and morbidity and generally poor treatment outcomes. ${ }^{[29]}$ Gene therapy for haemophilia offers a single administration of the therapeutic to potentially produce a lifelong cure and so overcomes many of the shortcomings of traditional replacement therapy. Several gene therapy programmes for both haemophilia A and B are ongoing and use hepatotropic AAVs to deliver the factor-encoding transgene to the liver. ${ }^{[30-32]}$ The results of both FVIII and FIX gene therapy have been encouraging, with many patients successfully converted from severe to moderate or mild phenotypes. At 8-year follow-up, there has been no loss of transgene expression or adverse events in the FIX gene therapy studies. Continued follow-up will help to establish whether expression will be lifelong.

Experience of haemophilia gene therapy in SA is increasing, with several FVIII and FIX programmes having started. Two SA patients who received gene therapy for severe haemophilia B in Europe are currently being followed up locally. Their FIX expression has remained stable in the absence of replacement therapy. A regional gene therapy infusion centre has been set up in Johannesburg, SA, and patients for several FVIII and FIX gene therapy studies are currently being recruited. Participation of patients of African origin in gene therapy is particularly important, as they have generally been underrepresented in global gene therapy studies. Eligibility for these studies requires the absence of antibodies against the viral vector used to deliver the gene therapy. Characterisation of the prevalence of antibodies against various AAV serotypes in Africa is currently underway. With high levels of poverty, inequality and a high disease burden, Africa will undoubtedly benefit from haemophilia gene therapy, as replacement therapy is currently unaffordable and inaccessible to the majority of patients in the region.

\section{Conclusion}

The question is often raised as to whether investment in technologies such as gene therapy is relevant to lower-to-middle income countries (LMICs). Basic healthcare, education and food and water security are usually considered more pressing priorities. In other words, can investment in sophisticated technologies be justified in the face of an inability to meet basic needs? Our view is that the need in LMICs is as great, if not greater, as in high-income countries, where most of the research on gene therapy is performed. ${ }^{[7]}$ This opinion is based largely on the promising expected clinical outcomes and principles of health economics. Little has been done to estimate the benefits to LMICs that would accrue from large-scale exploitation of new technologies arising from developments of the genomics era (including gene therapy). For example, the cost of living with HIV needs to be assessed against that of a possibly expensive single therapeutic procedure. Administering lifelong antiretroviral therapy, treatment of complicating infections and cancer, and the financial consequences that result from illness of economically active individuals collectively place a high burden on society. Administering one-off curative therapeutic procedures, such as those which may be possible with gene therapy, is therefore attractive. Although estimated costs of gene therapy are high, bringing this technology into mainstream practice is likely to reduce costs as economies of scale come to bear. In addition to investment in these technologies, our country also needs to develop the requisite regulatory and legislative frameworks to manage gene and cell therapeutics that will eventually enter the SA market. ${ }^{[33,34]}$

Significant progress in gene and cell therapy has been made over the past few decades, with a substantial increase in the number of 
studies that have progressed to clinical trials. This has translated to a number of therapeutics entering the market, with the first such drug, Gendicine, having been licensed in China for the treatment of head and neck tumours since 2003. Glybera and Strimvelis were approved by the European Medicines Agency in 2012 and 2016, respectively. Since 2015, the FDA has approved licenses for four gene-based therapeutics (Imlygic, Kymriah, Luxturna and Yescarta), with Kymriah and Yescarta also having been approved for commercialisation in Europe. It is clear that gene and cell therapy holds significant promise to address unmet medical needs. Advances in the field will soon see gene therapy taking its place at the forefront of medical science. It is important that SA takes the necessary steps to reap the benefits of these promising technologies.

\section{Acknowledgements. None.}

Author contributions. The authors contributed equally to the article. AE compiled the final manuscript.

Funding. The authors gratefully acknowledge funding from the SAMRC, the National Research Foundation and the Poliomyelitis Research Foundation.

Conflicts of interest. None.

1. Osterman JV, Waddell A, Aposhian HV. Gene therapy systems: The need, experimental approach, Osterman J, Waddell A, Aposhian HV. Gene therapy systems: The need, experimental approach,
and implications. Ann N Y Acad Sci 1971;179:514-519. https://doi.org/10.1111/j.1749-6632.1971. and implicatic

2. Friedmann T, Roblin R. Gene therapy for human genetic disease? Science 1972;175(4025):949-955. https://doi.org/10.1126/science.175.4025.949

3. Terheggen HG, Lowenthal A, Lavinha F, Colombo JP, Rogers S. Unsuccessful trial of gene replacement in arginase deficiency. Z Kinderheilkd 1975;119(1):1-3. https://doi.org/10.1007/bfo0464689

4. Friedmann T. Stanfield Rogers: Insights into virus vectors and failure of an early gene therapy model. Mol Ther 2001;4(4):285-288. https://doi.org/10.1006/mthe.2001.0454

5. Blaese RM, Culver KW, Miller AD, et al. Tlymphocyte-directed gene therapy for ADA-SCID: Initial trial results after 4 years. Science 1995;270(5235):475-480. https://doi.org/10.1126/science.270.5235.475

6. Marshall E. Gene therapy death prompts review of adenovirus vector. Science 1999;286(5448):22442245. https://doi.org/10.1126/science.286.5448.2244

7. Ginn SL, Amaya AK, Alexander IE, Edelstein M, Abedi MR. Gene therapy clinical trials worldwide to 2017: An update. J Gene Med 2018;20(5):e3015. https://doi.org/10.1002/jgm.3015 8. United States Food and Drug Administration. Approved cellular and gene therapy products. https://
www.fda.gov/BiologicsBloodVaccines/CellularGeneTherapyProducts/ApprovedProducts/default.htm (accessed 12 October 2018)

9. Maepa MB, Ely A, Grayson W, Arbuthnot P. Sustained inhibition of HBV replication in vivo after systemic injection of AAVs encoding artificial antiviral primary microRNAs. Mol Ther Nucleic Acids 2017;7:190-199. https://doi.org/10.1016/j.omtn.2017.04.007

10. Scott T, Moyo B, Nicholson S, et al. ssAAVs containing cassettes encoding SaCas 9 and guides targeting hepatitis B virus inactivate replication of the virus in cultured cells. Sci Rep 2017;7(1):7401. https://doi. org/10.1038/s41598-017-07642-6
11. Felgner PL, Gadek TR, Holm M, et al. Lipofection: A highly efficient, lipid-mediated DNAtransfection procedure. Proc Natl Acad Sci USA 1987;84(21):7413-7417. https://doi.org/10.1073 transfection pro
pnas. 84.21 .7413

12. Gorle S, Ariatti M, Singh M. Novel serum-tolerant lipoplexes target the folate receptor efficiently. Eur J Pharm Sci 2014;59:83-93. https://doi.org/10.1016/j.ejps.2014.04.012

13. Habib S, Singh M, Ariatti M. Glycosylated liposomes with proton sponge capacity: Novel hepatocyte-specific gene carriers. Curr Drug Deliv 2013;10(6):685-695. https://doi.org/10.2174/1 5672018113109990042

14. Mbatha L, Chakravorty S, De Koning $\mathrm{CB}$, et al. Spacer length: A determining factor in the design of galactosyl ligands for hepatoma cell-specific liposomal gene delivery. Curr Drug Deliv 2016;13(6):935-945. https://doi.org/10.2174/1567201813666160224123450

15. Barmania F, Pepper MS. C-C chemokine receptor type five (CCR5): An emerging target for the control of HIV infection. Appl Transl Genom 2013;2:3-16. https://doi.org/10.1016/j.atg.2013.05.004

16. Myburgh R, Cherpin O, Schlaepfer E, et al. Optimization of critical hairpin features allows miRNAbased gene knockdown upon single-copy transduction. Mol Ther Nucleic Acids 2014;3:e207. https:/ doi.org/10.1038/mtna.2014.58

17. Myburgh R, Ivic S, Pepper MS, et al. Lentivector knockdown of CCR5 in hematopoietic stem and progenitor cells confers functional and persistent HIV-1 resistance in humanized mice. J Virol 2015;89(13):6761-6772. https://doi.org/10.1128/JVI.00277-15

18. Jackson CS, Durandt C, Janse van Rensburg I, Praloran V, Brunet de la Grange P, Pepper MS. Jackson CS, Durandt C, Janse van Rensburg I, Praloran V, Brunet de la Grange P, Pepper MS.
Targeting the aryl hydrocarbon receptor nuclear translocator complex with DMOG and Stemregenin Targeting the aryl hydrocarbon receptor nuclear translocator complex with DMOG and Stemregenin
1 improves primitive hematopoietic stem cell expansion. Stem Cell Res 2017;21:124-131. https://doi. $1 \mathrm{improves}$ primitive hematop
org/10.1016/j.scr.2017.04.007

19. World Health Organization. Global hepatitis report, 2017. Geneva: WHO, 2017. http://www.who. int/hepatitis/publications/global-hepatitis-report2017/en/ (accessed 12 October 2018).

20. Carmona S, Ely A, Crowther C, et al. Effective inhibition of HBV replication in vivo by anti-HB short hairpin RNAs. Mol Ther 2006;13(2):411-421. https://doi.org/10.1016/j.ymthe.2005.10.013

21. Ely A, Naidoo T, Arbuthnot P. Efficient silencing of gene expression with modular trimeric Pol I expression cassettes comprising microRNA shuttles. Nucleic Acids Res 2009;37(13):e91. https://doi. org/10.1093/nar/gkp446

22. Marimani MD, Ely A, Buff MC, et al. Inhibition of replication of hepatitis B virus in transgenic mice following administration of hepatotropic lipoplexes containing guanidinopropyl-modified siRNAs. J Control Release 2015;209:198-206. https://doi.org/10.1016/j.jconrel.2015.04.042

23. Bloom K, Ely A, Mussolino C, Cathomen T, Arbuthnot P. Inactivation of hepatitis B virus replication in cultured cells and in vivo with engineered transcription activator-like effector nucleases. Mol Ther 2013;21(10):1889-1897. https://doi.org/10.1038/mt.2013.170

24. Crowther C, Ely A, Hornby J, et al. Efficient inhibition of hepatitis B virus replication in vivo, using polyethylene glycol-modified adenovirus vectors. Hum Gene Ther 2008;19(11):1325-1331. https:// doi.org/10.1089/hgt.2008.066

25. Ivacik D, Ely A, Ferry N, Arbuthnot P. Sustained inhibition of hepatitis B virus replication in vivo using RNAi-activating lentiviruses. Gene Ther 2015;22(2):163-171. https://doi.org/10.1038/ gt.2014.94

26. Giannelli F, Green PM. The molecular basis of haemophilia A and B. Baillieres Clin Haematol 1996;9(2):211-228. https://doi.org/10.1016/s0950-3536(96)80059-x

27. Srivastava A, Brewer AK, Mauser-Bunschoten EP, et al. Guidelines for the management of hemophilia. Haemophilia 2012;19(1):e1-47. https://doi.org/10.1111/j.1365-2516.2012.02909.X

28. Reding MT. Immunological aspects of inhibitor development. Haemophilia 2006;12 Suppl 6:30-35 discussion 35-36. https://doi.org/10.1111/j.1365-2516.2006.01363.x

29. Astermark J, Lacroix-Desmazes S, Reding MT. Inhibitor development. Haemophilia 2008;14 Suppl 3:36-42. https://doi.org/10.1111/j.1365-2516.2008.01711.x

30. George LA, Sullivan SK, Giermasz A, et al. Hemophilia B gene therapy with a high-specific-activity factor IX variant. N Engl J Med 2017;377(23):2215-2227. https://doi.org/10.1056/NEJMoa1708538

31. Miesbach W, Meijer K, Coppens M, et al. Gene therapy with adeno-associated virus vector 5-human factor IX in adults with hemophilia B. Blood 2018;131(9):1022-1031. https://doi.org/10.1182/
for blood-2017-09-804419

32. Rangarajan S, Walsh L, Lester W, et al. AAV5-Factor VIII gene transfer in severe hemophilia A. N Engl J Med 2017;377(26):2519-2530. https://doi.org/10.1056/NEJMoa1708483

33. Pepper MS, Pope A, Kling S, Alessandrini M, Green RJ. Ethical considerations in the application of cell and gene therapies in children. S Afr Med J 2018;108(12):1027-1029.

34. Pepper MS, Alessandrini M, Pope A, Van Staden W, Green RJ. Cell and gene therapies at the forefront of innovative medical care: Implications for South Africa. S Afr Med J 2019;109(1):20-22. 